\title{
Una literatura anfibia
}

An Amphibious Literature

Uma literatura anfíbia

\section{Silviano Santiago}

UNIVERSIDADE FEDERAL FLUMINENSE, BRASIL

Profesor jubilado de la Universidade Federal Fluminense, Niteroi, en

Brasil. Doctor en Letras por la Universidad de París, Sorbonne.

Ha publicado varios ensayos, entre otros Vale quanto pesa;

ensaios sobre questões político-culturais (1982), Nas malhas

da letra (1989), O cosmopolitismo do pobre: crítica literária

e crítica cultural (2004) y As raízes e o labirinto da América

Latina (2006). Correo electrónico: silviano@bighost.com.br

\author{
Artículo de reflexión \\ Documento accesible en línea desde la siguiente dirección: http://revistas.javeriana.edu.co \\ Trabajo leído el día 19 de abril de 2002, en John F. Kennedy Library (Boston), con motivo del homenaje realizado \\ al Premio Nobel José Saramago ("Tribute to José Saramago"). Publicado en portugués en la revista Alceu 3.5 \\ (julio-diciembre, 2002): 13-21. Difundido en medio impreso en Río de Janeiro por PUC-RJ. Expresamos \\ nuestro agradecimiento a Silviano Santiago por su amabilidad para posibilitar y acompañar esta traducción. \\ Traducción al español por Carolina Villada Castro, Universidad de Antioquia. \\ Correo electrónico: carolina.villadacastro@gmail.com
}




\section{Resumen}

En Brasil, la literatura es un campo en el que la estética — su componente cosmopolita- es inseparable de la política — su componente nacional-. La naturaleza híbrida, o anfibia, de la literatura brasilera contemporánea puede tener como efecto la alienación de los lectores tanto en el país como en el extranjero. Para los escritores literarios, la ficción constituye simultáneamente la producción y la diseminación del conocimiento, por medio de principios tomados de la vanguardia. Este doble proyecto solo es consumido por unos pocos en Brasil, en virtud de la alta tasa de analfabetismo. Programas de televisión muchas veces sustituyen la lectura. El doble proyecto también encuentra pocos entusiastas en el extranjero, ya que los lectores de primer mundo tienden a privilegiar los textos exportados que denuncian las injusticias sociales por medio del uso de una retórica sentimental, muchas veces demagógica.

Palabras clave: literatura y política; literatura brasilera contemporánea; retórica sentimental y demagógica

\section{Abstract}

In Brazil, literary art is a realm in which esthetics - its cosmopolitan component- is inseparable from politics - its local component-. This mixed, or amphibious, nature of contemporary Brazilian literature can have the effect of alienating readers both domestically and abroad. For literary artists, fiction constitutes simultaneously the production and dissemination of knowledge. This dual project is only partially attained in Brazil, where book shows on television have in many instances replaced reading, and internationally, where foreign readers have tended to prefer texts produced for export by lesser Brazilian writers, who denounce social injustice through the use of shocking rhetoric. This presentation addresses the difficulties that writers face in their utopian quest to explore the liberating aspects of art and politics in a country and in a region in which economic inequality, violence and social alienation are defining traits of the social order.

Keywords: literature and politics; contemporary Brazilian literature; shocking rhetoric

\section{Resumo}

No Brasil, a literatura é um campo no que a estética — seu componente cosmopolita-é inseparável da política — seu componente nacional-. A natureza híbrida, ou anfíbia, da literatura brasileira contemporânea pode ter como efeito a alienação dos leitores tanto no país quanto no estrangeiro. Para os escritores literários, a ficção constitui simultaneamente a produção e disseminação do conhecimento, através de princípios tirados da vanguarda. Este projeto duplo apenas é consumido por uns poucos no Brasil, em virtude da alta taxa de analfabetismo. Programas de televisão muitas vezes substituem a leitura. $\mathrm{O}$ projeto duplo também encontra poucos entusiastas no exterior, pois os leitores do primeiro mundo tendem a privilegiar os textos exportados denunciando injustiças sociais por meio do uso de uma retórica sentimental, muitas vezes demagógica.

Palavras-chave: literatura e política; literatura brasileira contemporânea; retórica sentimental e demagógica 
"Amphibious adj. [Gr. amphibios, living a double life; amphi-, on both sides + bios, life. ... 3. having two natures or qualities; of a mixed nature". WEBSTER'S DICTIONARY

VENGO DE UN país en el que un segmento considerable de la población aún está compuesta de analfabetas. Eso trae consecuencias para la literatura y las artes producidas allí. Nosotros, escritores, hemos considerado que la publicación en libro de las obras literarias que imaginamos, es tan importante como la acción persuasiva que ese libro puede ejercer en el plano político, en el caso de que sea leído por el restringido grupo social letrado que lo consume, o que se lo anuncie o se lo comente por los medios de comunicación masivos. A falta de una mejor explicación descriptiva, me valgo de una metáfora: nuestro sistema literario se asemeja a un río subterráneo, que corre desde la fuente hasta la desembocadura, sin tocar las márgenes que, sin embargo, lo conforman.

Otra consecuencia del analfabetismo que se propaga entre los desfavorecidos, esta vez asociada al extraordinario éxito de los medios digitales, trasciende el campo propiamente literario. De la noche a la mañana, el escritor se transforma en intelectual responsable. Alcanza al público que su libro no tiene. El mayor drama del analfabetismo en Brasil es el de haber servido de adorno para los medios digitales, con el consiguiente desarraigo de la prensa escrita. El brasilero aprendió a escuchar radio y a ver televisión; pocos saben o quieren leer. Esa afirmación desconcertante no recubre solo la camada de desfavorecidos: se volvió consenso nacional a partir de la dictadura militar de 1964 .

Si en un país de más de 150 millones de habitantes ${ }^{1}$ es bajísima la tasa de consumo per capita del libro, ya el habla de quien ejerce el oficio literario puede ser sintonizada sin graves obstáculos en los medios digitales - en especial en la televisión educativa y en la televisión por cable, pero no exclusivamente- Concedida a los pares de los medios televisivos, muchas veces la entrevista sirve al escritor de trampolín para discusiones públicas sobre ideas implícitas en la obra

1 Nota de la traductora: en 2015 Brasil tenía más de 200 millones de habitantes. 
literaria. El libro es raramente apreciado por la lectura. Se consume la imagen del intelectual, se asimilan sus ideas, por más complejas que sean. Estas derivan en una energía motriz de bajísimo voltaje, que impele al televidente común a enfrentar los problemas nacionales, obligado a apoyarse apenas no más que en las amarguras de lo cotidiano como fundamento para la revuelta. Por otro lado, hay un peligroso culto a la personalidad que ronda al escritor aprendiz. Muchos jóvenes se sienten tan contentos con la imagen pública de intelectual, que luego se despreocupan de la artesanía literaria, o la abandonan de una vez por todas.

Si las márgenes del río metafórico, al que nos referimos arriba, pasan a lo largo del libro, acaban por aproximarse indirectamente a él a través de la entrevista dada por el autor. Aquella es el modo que el escritor encontró para poder comunicarse con un público más amplio sin perder los privilegios excluyentes del oficio literario que escogió. Al contrario de lo que sucede en sociedades con mayor tasa de alfabetización y escolaridad, en Brasil el libro de buena calidad puede ser el motivo de la entrevista mediática, mas nunca es su fin. En otras palabras, la programación de libros en venta de buena calidad [en el Brasil] no pasa, o pasa muy poco, por los medios digitales. En compensación, ideas de tenor revolucionario, en virtud de la entrevista con escritores de buen nivel, circulan con más frecuencia entre televidentes brasileros que entre televidentes del primer mundo.

Libro de entrevista, hoja de papel y pantalla; escrito y habla —estamos ante situaciones concretas excluyentes, que se dan como cómplices para el escritor doble del intelectual, e irreconciliables para la mayoría de la población-.

Con el paso de las décadas, la práctica de la literatura en Brasil se fue revistiendo de un manto, o sea, de una doble meta ideológica. $\mathrm{Al}$ aprovechar los embrollos de la observación directa de los acontecimientos cotidianos o históricos y al incentivar la reflexión sobre los observadores privilegiados, nuestra literatura configura la carencia socio-económica y educativa de la mayoría de la población del país así como define, por el ejercicio impiedoso de la auto-crítica, el grupo reducido y singular que ha asumido de uno u otro modo las formas clásicas de mando y gobernabilidad en las naciones de América Latina.

Por un lado, el trabajo literario busca dramatizar objetivamente la necesidad del rescate de los miserables con el fin de elevarlos a la condición de seres humanos (ya no digo a condición de ciudadanos) y, por otro lado, procura avanzar - por la elección de personas del círculo social de los autores como personajes de la literatura- con un análisis de la burguesía económica en sus desaciertos e injusticias seculares. De ese doble y contradictorio énfasis ideológico - del que los escritores no consiguen desvincularse en virtud del papel que, como vimos, 
aún ocupan en la esfera pública de la sociedad brasilera - adviene el carácter anfibio de nuestra producción artística.

En el siglo XX, nuestros mejores libros apuntan hacia el arte, al observar los principios individualizadores, emancipadores y rigurosos de la vanguardia estética europea y, al mismo tiempo, apunta hacia la política, al querer denunciar por medio de los recursos literarios no solo las heridas oriundas del pasado colonial y esclavocrata (escravocrata $)^{2}$ de la sociedad brasilera, sino también los regímenes dictatoriales militares que destruyeron la vida republicana. La actividad artística del escritor no se desliga de su influencia política; la influencia de la política sobre el ciudadano no se desliga de su actividad artística. El todo se completa en una forma casi incompleta en apariencia, solo en apariencia. Al dramatizar los grandes problemas de la sociedad brasilera en el contexto global, y los impases que la nación atraviesa en el plano nacional, la literatura quiere, en una paradoja evidente, hablar en particular al ciudadano brasilero responsable. No son muchos, desgraciadamente.

Como consecuencia de aquel doble y contradictorio enfoque ideológico surge un vacío temático en nuestra literatura que, a mi modo de ver, acaba siendo ocupado por la gran cantidad de libros de literaturas extranjeras que son traducidos y consumidos en Brasil. Tenemos una industria editorial ágil y actualizada en un mercado del libro cosmopolita y guloso de novedades. En la singularidad de nuestra industria editorial y de nuestro mercado del libro están las dos razones que justifican la importancia que aún se da a la artesanía literaria entre escritores que, sin la afluencia masiva de las literaturas extranjeras, hace mucho habrían abandonado la pretensión de hacer arte. El vacío temático se refiere a la parca dramatización en la literatura de los problemas dominantes de la clase media, que queda agotada entre los dos extremos de la sociedad. La literatura brasilera hizo caricatura; ha pasado por encima de la complejidad existencial, social y económica de la pequeña burguesía, aguzando la perspicacia de su crítica en una configuración socioeconómica anticuada del país, semejante a la que nos fue heredada a finales del siglo XIX. Si el Brasil republicano alcanzó el progreso material, está muy lejos del progreso social. Los buenos escritores e intelectuales brasileros por lo demás son sensibles a esa desarmonía.

No es coincidencia que, en nuestra literatura, la clase media solo tenga conciencia de su situación específica bajo la forma de la decadencia social. No

2 Nota de la traductora: proponemos la traducción esclavo-crata más que esclavista, para enfatizar el carácter sistemático de una lógica de gobierno colonial de las poblaciones como sustrato histórico de las prácticas de gobierno contemporáneas. 
es coincidencia que el tema del declive de las grandes familias rurales recorra gran parte de nuestra literatura novecentista, llevando algunos críticos a tomar el título de la novela de Lúcio Cardoso - A crônica da casa assassinada $-{ }^{3}$ como metáfora y emblema del proceso constituyente de la clase media urbana en el país. Los ricos oligarcas son desposeídos del poder económico por la industrialización y transformados en funcionarios públicos o profesionales liberales por el Estado Nacional en busca de modernización, que encuentran en las calles de las metrópolis los ambiciosos extranjeros e hijos de extranjeros, firmes en promover el desarrollo del Brasil industrial. Ex-oligarcas e inmigrantes nuevos ricos, todos asociados directa o indirectamente al capital extranjero, acaban por mantener un matizado segmento medio en las grandes ciudades, infelizmente poco presente en nuestra mejor literatura.

Cuando trasciende las fronteras nacionales por la traducción para otros y diversos idiomas, el libro brasilero sale en busca de nuevos lectores, diferentes de los que fueron configurados por décadas de práctica literaria espuria y legítima. El carácter anfibio de nuestra producción artística puede parecer - y muchas veces parece- poco seductor a los ojos exigentes del ciudadano del mundo. La mirada cosmopolita se relaciona con el libro por medio de la notable tradición literaria occidental; por medio de la percepción política de la realidad nacional en la que se inserta el brasilero, y de la realidad global en la que todos terminamos por integrarnos.

El público extranjero (y por público extranjero estoy tratando de caracterizar, tal vez de manera torpe, a los lectores que viven en los países considerados como pertenecientes al primer mundo) acostumbra ser radical en su gusto artístico. Acostumbra ser radical en la elección del libro de literatura que va a comprar. Por eso es poco propenso a acatar, por un lado, la discusión política en la estética y, por otro, los rodeos estéticos en la política. El lector extranjero cosmopolita, repetimos, acostumbra ser radical en su definición de los campos disciplinarios.

En su generoso radicalismo, el lector extranjero ha sido doblemente infeliz en la aprobación de la producción literaria brasilera. Desprecia a priori las obras que se definen por su carácter anfibio. No sirven ni de ejemplo de arte ni de política. Opta por desarticular los elementos ambivalentes, constituyentes de la duplicidad ideológica y temática de la literatura brasilera, en elementos aislados, autónomos, con vida propia. O arte, o política —-define la dirección

3 Nota de la traductora: novela del escritor brasilero Lúcio Cardoso, publicada en 1973, sobre el declive de las familias aristócratas, propietarias de grandes haciendas rurales en los albores de la industrialización/modernización brasilera. 
de interés a la hora de comprar. Nunca las dos al mismo tiempo o en el mismo lugar. Arte y política-. El híbrido le parece un fantasma. Fantasma que sin duda lo sorprenderá - en caso de que sea menos respetuoso de las fronteras nacionales y de las convenciones disciplinarias - en su propia cotidianidad de habitante del primer mundo. Como en Hamlet, el fantasma del híbrido puede susurrarle al oído que "the time is out of joint: Oh cursed spight, / That ever I was borne to set it right" [El mundo está fuera de sus ejes. ¡Oh! maldita suerte... / ¿por qué nací para ponerlo en orden?].

El lector extranjero no quiere comprender las razones por las que, en la literatura brasilera, lo legítimo quiere ser espurio con el objetivo de que lo espurio, a su vez, pueda ser legítimo. Su deseo de lector no se funda en el impulso del texto literario. Quiere distanciarse de él. Quiere percibir lo estético en el arte y lo político en la política. Quiere lo que el texto no quiere. No desea el texto que no lo desea. Cada mico en su palo (Cada macaco em seu galho), ${ }_{4}^{4}$ como dice el dicho. No comprende que el doble movimiento de impureza que se encuentra en la buena literatura brasilera no es razón para quejas estetizantes y mucho menos para críticas pragmáticas. La impureza es, por el contrario, la forma literaria por la que la lucidez se afirma doblemente. La forma literaria anfibia requiere la lucidez del creador y también la del lector, ambos impregnados por la condición precaria de ciudadanos en una nación dominada por la injusticia.

Por un lado, el lector extranjero tiende a buscar entre los libros de literatura que pretende leer aquellos que denuncian impúdicamente la condición miserable de gran parte de la población brasilera. Generalmente son libros de literatura que poco se preocupan de satisfacer los mínimos requisitos que transformarían en forma de arte el hecho bruto socio-económico. Están más próximos del reportaje periodístico (no confundirlo con el lenguaje periodístico en la buena literatura, que puede ser un recurso estilístico notable) que de la literatura. La brutalidad en sí de lo material representado es motivo para el interés sentimental por el Brasil (país de las desgracias humanas y de las catástrofes civiles); para la admiración por el escritor (el coraje y la audacia en la denuncia) y los elogios desgarrados al libro.

No nos hagamos ilusiones: la brutalidad desnuda y cruel también es motivo para la compasión del lector extranjero. Ante todo, el lector extranjero ha sido

4 Nota de la traductora: expresión popular brasilera que indica que cada persona tiene que permanecer en su lugar, ocupándose de lo que le concierne. Recuerda también el título y motivo de la canción del sambista Bahiano Riachão, grabada por Gilberto Gil y Caetano Veloso en 1972, al regreso del exilio político en Londres durante la dictadura militar en Brasil. 
un ser de sentimientos cristianos. Eso es bueno. Eso pasa a ser malo cuando confunde los buenos sentimientos con una mezcla de altruismo abstracto y filantropía remota, o cuando se deja confundir por la propia bondad, sintiéndose más distraído de los llamados del mundo y menos deplorable en su confort. Se siente de tal modo inmerso en las aguas excitantes de la denuncia explotada por el libro, que se olvida - durante el proceso de lectura - de reflexionar sobre su papel, por mínimo que sea, en esa historia que, en el caso de que hubiera sido dramatizada con recursos artísticos menos lamentables, podría haber colocado a la hipocresía contra la pared. El lector de buenos sentimientos se alimenta de la brutalidad que le es transmitida y pierde el norte de sí mismo con su contundencia. La brutalidad es el territorio en el que los buenos sentimientos del lector exorcizan el hechizo armado por Otro subdesarrollado. Ella no hace parte de su dura realidad de todos los días. Si lo hace, prefiere divisarla con binóculos: allá lejos, entre las carátulas del libro, en un país marginal. Cerrado el libro, los buenos sentimientos exhalan el último suspiro.

Por otro lado, hay entre nosotros, escritores que son indiferentes a la doble llamada ideológica a la que nos referimos al inicio. Curiosamente, es por la indiferencia a la miseria nacional que llegan a encontrar un público cautivo en el extranjero. No hay cómo criticar a los compañeros de letras que optan por el camino de la pureza artística en un país en donde, por este o aquel motivo, no tendrían los libros comprados por los coterráneos. Se visten de anacoretas o ascetas. Se sienten tentados por la cruel realidad que los asombra al doblar cada esquina y buscan, entre tanto, la pureza artística. Quieren la reencarnación, en la obra literaria que realizan a duras penas, y las pocas recompensas financieras de una ética platónica (el bien, lo bueno, la luz...).

El lector extranjero, en su radicalismo disciplinario, tiende a comprar y leer -para complementar la obra exclusivamente política, algunas veces de tenor demagógico- la obra literaria pura. Esta dramatiza los pequeños grandes dramas humanos con rigor estilístico y delicadeza psicológica. En su universalismo y aristocratismo confesos, esa obra está desprovista de cualquier vínculo originario con la cultura en la que brota. Trasciende territorios geográficos para instalarse en la eternidad del trabajo artístico. Una complicidad de sensibilidad y casta une al autor brasilero y al lector extranjero por intermedio del ejercicio de la lectura del libro totalmente comprometido con los valores fuertes y tradicionales de la literatura occidental.

En la obra de literatura pura brasilera está representado, bajo la forma de espejo, el retrato de Dorian Gray. La estilización literaria no torna más agudos los problemas de la representación; por el contrario los omite con una hipnosis 
de magia artística. El compromiso con el Tiempo, con mayúscula, mutila los disparates del tiempo, con minúscula. La eternidad se hace escrita y la escritura literaria se hace atemporal.

Por desconocer a su otro - la política nacional—, el territorio específicamente literario es amplio y no tiene fronteras estilísticas ni barreras ideológicas. Escritores brasileros y extranjeros, lectores brasileros y extranjeros - viven todos en una comunidad de elegidos, en la que predomina la pureza de los principios y valores artísticos-. La obra de arte es objeto de conmoción y deleite para los iniciados. Eso no es malo para la literatura brasilera: una literatura que se quiere tan contemporánea, nuestra y sofisticada como las demás literaturas de esta parte del mundo al que pertenecemos. Eso no es malo para los escritores que, habiendo optado por lo híbrido, nunca se descuidan del eterno aprendizaje del oficio literario.

¿Habremos llegado a un callejón sin salida? ¿A un desencuentro entre el libro de buena calidad brasilero y el lector extranjero? ${ }_{\mathrm{C}}$ Deberíamos quedar cada uno por su lado, ya que las idiosincrasias de un grupo contrastan con las idiosincrasias de otro grupo? No creo. Ante todo, es para eso que existen estos encuentros culturales, en los que territorios y banderas nacionales son puestos de lado, y no abandonados, con el fin de que nos encaminemos en dirección del entendimiento que se pretende común. Se busca conocer mejor una forma de saber particular - el saber literario en su forma brasilera-, para que funcione al mismo tiempo como la marca de que cada uno de nosotros busca el conocimiento universal al perderse a sí mismo para reencontrarse en el lenguaje y en la experiencia del Otro.

No se trata de ubicar a los participantes de este encuentro en la ciudad de Boston - que aquí están para debatir las singularidades de la literatura escritas en lengua portuguesa-; no se trata de ubicarnos, repito, en el optimismo que los grupos destinan a ellos por ser dominadores incuestionables. Por el contrario, se trata de resaltar un optimismo terco $(p e ́-d e-b o i),{ }^{5}$ para usar la expresión popular. Un optimismo rudo y arduo, cojo y corajudo, polémico, presto a ser construido por el uno y por el otro, por todos. Importa el proceso precario de construcción de la utopía. El edificio listo, tranquilo para ser habitado, solo existe en la tierra para los ricos y poderosos, y desde siempre en el reino de los cielos para todos los humanos.

En el caso analizado, el precario proceso de construcción de la utopía a la que nos estamos refiriendo, se verificará que esta no es tan diferente del proceso de construcción de la obra literaria, que venimos clasificando de anfibia, o híbrida.

5 Nota de la traductora: expresión popular brasilera que alude a las personas aferradas a una idea o cosa. 
No hay cómo no auto-clasificarse de visionario si usted es escritor en un país como Brasil. Visionario significa que usted tiene visiones - en este caso literarias y políticas-, que significan que la situación socio-económica y educativa del país no será para siempre la misma. Ella puede y va a mejorar.

El escritor brasilero tiene la visión del arte como forma de conocimiento, tan legítima como las formas de conocimiento de las que se sienten únicas poseedoras las ciencias exactas y las ciencias sociales y humanas. Él también tiene la visión política como ejercicio del arte que busca el bien y el justo gobierno de los pueblos, disociándola de la demagogia de los gobernantes; del populismo de los líderes carismáticos y de la fuerza militar de los que buscan el orden a hierro y fuego.

Arte y política se dan la mano en la literatura brasilera para decir que la educación, como nos exhortó a pensar Anísio Teixeira en el pasado 1957 (año en que ingresé a la Universidad Federal de Minas Gerais), no es un privilegio. Vale la pena re-leerlo en esta ocasión:

Cuando en la Convención Francesa, se formuló el ideal de una educación escolar para todos los ciudadanos, no se pensaba tanto en universalizar la escuela existente, sino en una nueva concepción de la sociedad, en la que los privilegios de clase, de dinero y de herencia no existieran, y el individuo pudiera buscar por la escuela su posición en la vida social.

En caso de que la educación no hubiera sido privilegio de pocos desde los tiempos coloniales, tal vez hubiéramos podido escribir de otra manera el panorama de la literatura brasilera contemporánea. Tal vez lo legítimo no hubiera sido la necesidad de buscar lo espurio para que aquello, a su turno, se tornara legítimo. Tal vez pudiéramos valernos apenas de dos principios de la estética: el libro de literatura existe $u t$ delectet y ut moveat (para deleitar y conmover). Pudiéramos atenernos a esos dos principios, y dejar de lado un tercer principio: ut doceat (para enseñar). Es esta, y no otra, la manera como nos toca narrarles en este día de primavera el panorama de la literatura brasilera contemporánea. 